\title{
Revision of a Press Fit Biological Fixation Stem Fracture in a Dog
}

\author{
Massimo Petazzoni $^{1} \quad$ Elena De Giacinto ${ }^{2}$ Denis Marcellin-Little ${ }^{3}$ Michael Kowaleski ${ }^{4}$ \\ ${ }^{1}$ Department of Orthopaedics and Traumatology, Clinica Veterinaria \\ Address for correspondence Elena De Giacinto, Giovanni Pascoli \\ Milano Sud, Borromeo, MI, Italy \\ ${ }^{2}$ Clinica Veterinaria San Carlo, Brescia, BS, Italy \\ 3 University of California Davis, Davis, California, United States \\ ${ }^{4}$ Department of Clinical Sciences, Cummings School of Veterinary \\ Medicine at Tufts University, North Grafton, Massachusetts, \\ United States
}

VCOT Open 2019;2:e35.

ERRATUM
It has been brought to the Publisher's attention that the Conflict of Interest statement was incorrect in the above article published in VCOT Open, Volume 2, Number 1, 2019 (DOI: 10.1055/s-0039-1683892). The correct statement appears as follows:

Conflict of Interest

Michael Kowaleski and Denis Marcellin-Little are clinical consultants and course instructors for BioMedtrix. Massimo Petazzoni designed the Fixin system and is a consultant for Intrauma, the company which owns patents on, produces, and sells the Fixin system. Michela Buiatti was an Intrauma consultant when the manuscript was written. 